Sprache und Raum 
Harro Schweizer (Hrsg.)

\section{Sprache und Raum}

Psychologische und linguistische Aspekte der Aneignung und Verarbeitung von Räumlichkeit

Ein Arbeitsbuch für das Lehren von Forschung

J.B. Metzlersche Verlagsbuchhandlung Stuttgart 


\section{CIP-Kurztitelaufnahme der Deutschen Bibliothek}

\section{Sprache und Raum :}

psycholog. u. linguist. Aspekte

d. Aneignung u. Verarb. von Räumlichkeit ;

e. Arbeitsbuch für d. Lehren von Forschung /

Harro Schweizer (Hrsg.).

- Stuttgart : Metzler, 1985.

ISBN 978-3-476-00549-6

NE: Schweizer, Harro [Hrsg.]

ISBN 978-3-476-00549-6

ISBN 978-3-476-03189-1 (eBook)

DOI 10.1007/978-3-476-03189-1

(c) Springer-Verlag GmbH Deutschland 1985

Ursprünglich erschienen bei J. B. Metzlersche Verlagsbuchhandlung und Carl Ernst Poeschel Verlag GmbH in Stuttgart 1985 


\section{Vorbemerkung}

Erst im Zuge einer nicht-linguistische Kontexte einbeziehenden Sprachwissenschaft ist das Problem von Sprache und Raum in dieser übergreifenden, linguistische Teilbereiche integrierenden Form für die Sprachforschung interessant geworden. Im vorliegenden Band kommen Wissenschaftler verschiedener Disziplinen zu Wort, insbesondere aber Linguisten und Psychologen (bzw. solche, die sich im Schnittbereich der beiden Fachrichtungen definieren) mit oft recht unterschiedlichen Interessen, Standpunkten und Arbeitsbereichen. Sie mögen Gewähr bieten für die hier angestrebte breite Diskussion. Was dem einen Teil der Leser eine Auseinandersetzung mit neuen Uberlegungen ermöglichen oder Anregungen für den laufenden Forschungsprozeß bedeuten kann, kann anderen einen neuen Einstieg in den Wirkungs- und Funktionszusammenhang von Sprache und sprachlichem Handeln bieten.

Insofern ist der Band als "Arbeitsbuch für das Lehren von Forschung» konzipiert - seine Zielgruppe sind Forschende, Lehrende und Lernende gleichermaßen; in nahezu allen Beiträgen wird auf offene Fragen und sich eröffnende Problemstellungen verwiesen und finden sich kritische Würdigungen des jeweils erreichten Standes der Forschung. Meine Bemühungen gelten der Verbindung von aktuellen Forschungsbeiträgen zu unterschiedlichen Theorie-, Gegenstands- und Empiriebereichen mit dem Gesichtspunkt der $>$ Lehrbarkeit` als einem ganz zentralen, jedoch oft vernachlässigten Beurteilungskriterium wissenschaftlichen Arbeitens.

Mein besonderer Dank gilt Helmut Richter, dem ich überhaupt mein Interesse für die hier behandelte Thematik verdanke und der mich zur Verwirklichung dieses Bandes ermutigt hat, sowie Horst Ebbinghaus, der mir über seine sorgfältigen Ubersetzungen hinaus ein ideenreicher und vertrauter Gesprächspartner war.

Berlin (West) und Okrug Gornij

im April 1983

H. Sch. 


\section{Inhaltsverzeichnis}

Vorbemerkung

Harro Schweizer

Ein-Leitung: Was bedeutet dem Menschen der Raum?

I. Raum als kognitives und sprachliches Problem

Roger M. Downs, David Stea

Kognitive Karten und Verhalten im Raum - Verfahren und

Resultate der kognitiven Kartographie

Charlotte Linde, William Labov

Die Erforschung von Sprache und Denken anhand von Raumkonfigurationen

II. Syntaktische und semantische Aspekte: zum Lexikon der Raumrepräsentation

Dieter Wunderlich

Raum, Zeit und das Lexikon

Peter Rolf Lutzeier

Sprachliche Vermittler von Räumlichkeit - Zur Syntax

und Semantik lokaler Präpositionen

J. Peter Denny

Was ist universal am raumdeiktischen Lexikon?

III. Die Bedeutung des sprachlichen und nicht-sprachlichen Handelns im Raum

Veronika Ehrich

Zur Linguistik und Psycholinguistik der sekundären

Raumdeixis 
VIII Inhaltsverzeichnis

Dirk Wegner

Der persönliche Raum als Modell nonverbaler Proxemik

IV. Das kognitive, interaktive und sprachliche

Raumkonzept bei Kindern

\section{Thomas Thiel}

Räumliches Denken und das Verständnis von Lokativen

beim Spracherwerb

Jürgen Weissenborn

»Ich weiß ja nicht von hier aus, wie weit es von dahinten aus ist - Makroräume in der kognitiven und sprachlichen

Entwicklung des Kindes

V. Die kulturgeschichtliche Aneignung des natürlichen und sozialen Raumes

Konrad Ehlich

Literarische Landschaft und deiktische Prozedur: Eichendorff

Joachim Gessinger, Wolfert von Rahden

Uber die Geselligkeit von Stachelschweinen - Ansichten einer kulturhistorisch-interpretativen Proxemik 